\title{
Experimental Thermodynamics of the Helix-Random Coil Transition. I. Influence of Polymer Concentration and Solvent Composition in the PBG-DCA-EDC System
}

\author{
THEODOR ACKERMANN and EBERHARD NEUMANN, Institut für \\ Physikalische Chemie der Universität, Münster (Westfalen), Germany
}

\begin{abstract}
Synopsis
The course of the reversible helix formation of poly ( $\gamma$-benzyl L-glutamate) (PBG) dissolved in a mixture of dichloroacetic acid (DCA) and 1,2-dichloroethane (EDC) was followed by measuring the heat capacity and the optical rotation of the system through the transition region. The results of these measurements indicate that the transition enthalpy $\Delta H$, the transition temperature $T_{c}$, and the Zimm-Bragg parameter $\sigma$ depend considerably on the PBG concentration as well as on the composition of the solvent. For the standard state of infinite dilution, however, a linear extrapolation of the measured $\Delta H$ values results in a standard value $\Delta H^{\circ}=950 \mathrm{cal} . / \mathrm{mole}$, independent of the solvent composition. The results of the calorimetric measurements are discussed in relationship to changes in optical rotation. Some peculiarities in the measured thermodynamic and optical properties in solutions with relatively high content of dichloroacetic acid are reported.
\end{abstract}

\section{Introduction}

The proteins and the nucleic acids display in solution a secondary structure which depends on the external physical conditions. Changes in the secondary structure of these biopolymers often occur during many biochemical processes. A simple model reaction for such a structural change is the reversible helix-to-random coil transition of synthetic polypeptides.

There are several possible causes for a conformational transformation of this cooperative type, for example, variations in the temperature. ${ }^{2}$ The course of the cooperative helix-coil transition of a polypeptide can be conveniently followed by measuring the optical rotation as a function of temperature. $^{3}$

Recently, the experimental investigations concerning biopolymers have been widened by a new method. Nearly at the same time various groups of authors ${ }^{4-8}$ succeeded in gaining information on the thermal behavior and on the thermodynamics of dissolved polypeptides and nucleic acids in a direct way. The accurate measurement of the temperature course of the heat capacity through the transition region opens a way for determining directly the transition enthalpy $\Delta H$ for the solvated biopolymer, and, in 
addition, in the case of polypeptides, the cooperative parameter $\sigma .^{8}$ In the statistical model developed by Zimm and Bragg ${ }^{9}$ the term $\sigma$ is defined as a key parameter, which now is experimentally accessible by a calorimetric method.

The synthetic polypeptide poly( $\gamma$-benzyl L-glutamate) (PBG) is considered to be one of the best investigated biopolymers. In a solvent mixture of dichloroacetic acid (DCA) and 1,2-dichloroethane (EDC) this polypeptide is able to display an inverse helix-coil transition. When the temperature of such a PBG solution is increased, a structural change is observed from the randomly coiled form to the helical conformation within a relatively small temperature region. ${ }^{2}$ This transition is associated with an additional change in the enthalpy of the system. At constant composition of the solvent, both the transition enthalpy $\Delta H$ and the cooperative parameter $\sigma$ depend remarkably on the polymer concentration. ${ }^{4,8}$

Some orienting measurements at constant PBG concentration carried out by Karasz and $O^{\prime} R$ illy ${ }^{10}$ gave rise to the suggestion that different values of the transition enthalpy $\Delta H$ will be found in calorimetric measurements on PBG solutions having unequal weight fractions of dichloroacetic acid. Recently, this suggestion has been verified for the deuterated PBG system at two different compositions of the solvent. ${ }^{11}$ It may then be assumed that, only at relatively high PBG concentrations, a variation of the solvent composition would considerably affect the magnitude of $\Delta H$.

It is shown in the experimental part of this paper that the influence of the PBG concentration on the measured values of $\Delta H$ and $\sigma$ is superimposed by a remarkably strong dependence on the DCA content in the mixture.

An influence of polymer concentration and solvent composition on $\Delta H$ and on $\sigma$ has not been considered in the Zimm-Bragg theory and in the theoretical extensions of this treatment developed by Applequist. ${ }^{12}$

It was the purpose of this investigation to test whether the simple basic relations resulting from this model theory of the helix-coil transition remain applicable over wide limits of polymer concentration and solvent composition of the investigated system provided that the dependence of the thermodynamic parameters on the mole fractions of the components is taken into account.

\section{Materials}

Poly( $\gamma$-benzyl L-glutamate) (molecular weight 350,000) was purchased from l'ilot Chemicals. The dry product was dissolved in a prepared mixture of DCA and EDC (both from Fluka, Switzerland). The DCA was redistilled several times in vacuum, while the EDC was used without further purification. The purity of the solvents was tested by measuring the refractive index.

The concentration of PBG ( $c_{P B G}$ ) is expressed in moles of the amino acid residue dissolved in $1 \mathrm{~kg}$. of the solvent. The portions of the components in the solvent mixture are given in weight per cent, for example \$3 wt.-\% DCA. 


\section{Calorimetric Measurements}

The calorimetric measurements were carried out with a recording adiabatic calorimeter. A relatively complicated arrangement of mechanic and electronic instruments allows the measurement of the heat capacity by steps with the required high accuracy during continuous electrical heating of the calorimeter system. The calorimeter vessel has an internal volume of about $300 \mathrm{ml}$. It is furnished with electric heater rods and with a platinum-resistance thermometer. A general description of this precision apparatus is given elsewhere..$^{8,13,14}$ A heating rate of about $0.05^{\circ} \mathrm{C} . / \mathrm{min}$. was used.

A linear dependence of the measured heat capacity on temperature is obtained in each case for the pure solvent mixture of DCA and EDC without the polymer. The measurement of the heat capacity as a function of temperature leads for the PBG solutions to calorimetric transition curves which are characterized by a maximum of the additional heat capacity, $C_{c, \max }$ (see Fig. 6, ref. 8, and Figs. 1 and 2 of this paper), and the temperature range in which the cooperative conversion occurs. The temperature corresponding to $C_{c, \max }$ is called $T_{c}$, originally in the polarimetric transition curve defined as the midpoint of the conformational conversion. The calorimetric transition temperature is-at least in principle-not the temperature value for the maximum of the measured total heat capacity within the transition region. The maximum value of the additional heat capacity (observed minus base line) is defined as ${ }^{8}$

$$
C_{c, \max }=\Delta H(d \theta / d T)_{T_{c}}
$$

where $\theta$ is the fractional helix content of the polypeptide. The temperature dependence of $\theta$ at $T_{c}$ can be expressed in terms of the statistical treatments of Zimm and Bragg ${ }^{9}$ and of Applequist. ${ }^{12}$ From an approximative relation between $\theta$ and $T$, as discussed by Applequist, we get eq. (2):

$$
C_{c, \max }=(\Delta H)^{2} / 4 R T_{c}^{2} \cdot \sigma^{1 / 2}
$$

where $R$ is the gas constant and $T_{c}$ is the transition temperature Kelvin.

The transition enthalpy $\Delta H$, i.e., the additional molar enthalpy change of the system, is directly proportional to the area under the peak limited by the measured heat capacity data and the dashed baseline (corresponding to the hypothetical limiting case $\Delta H=0$ ); see Figures 1 and 2 .

The course of the heat capacity has been measured for solutions of different PBG concentrations at various DCA-EDC solvent compositions.

The calorimetric transition curves presented in Figure 1 show the transition temperature $T_{c}$ to be dependent on the polymer concentration at constant solvent composition. The slopes of the heat capacity versus $T$ lines below and above the transition region are not equal. This is especially marked for solutions with low concentrations of PBG.

The effect of the solvent composition on the transformation behavior of the system is demonstrated in Figure 2 for two solutions of equal PBG 


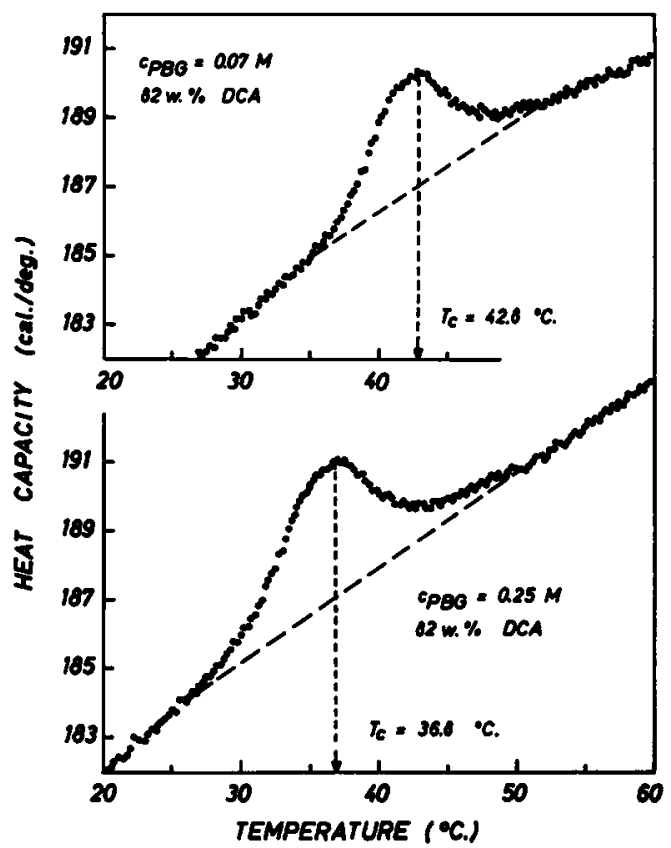

Fig. 1. Heat capacity as a function of temperature of two PBG solutions of different concentration, at constant solvent composition.

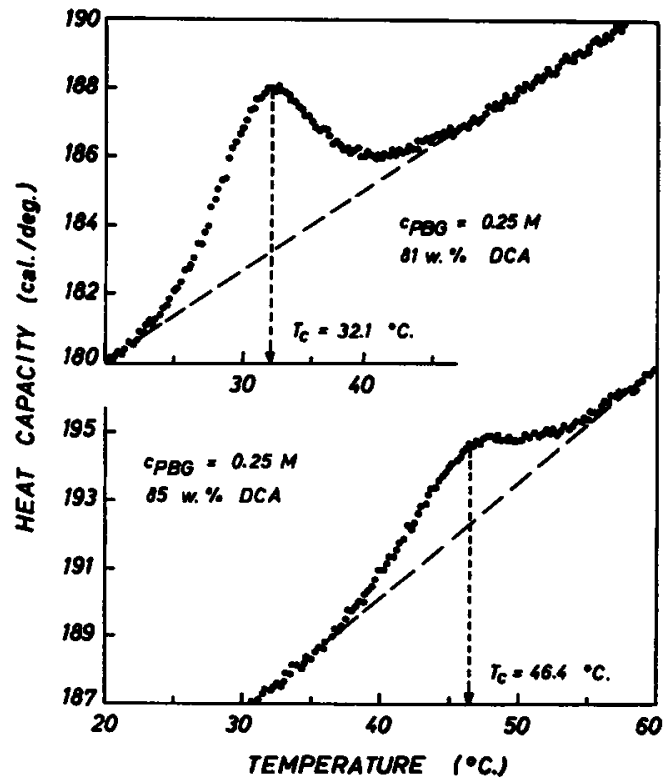

Fig. 2. Heat capacity as a function of temperature of two PBG solutions of equal concentration and of different DCA content. 
concentration. Some differences in the transition curves are clearly to be seen. For the solution of $85 \mathrm{wt} .-\%$ DCA the transition region has been shifted to higher temperatures, the transition temperature $T_{c}$ has a higher value, and the area under the peak is obviously smaller than that obtained for the solution of only $81 \mathrm{wt} . \%$ DCA.

\section{Polarimetric Measurements}

The polarimetric measurements were carried out with PBG solutions of solvent compositions corresponding to those calorimetrically employed. The optical rotation at $589 \mathrm{~m} \mu$ was measured as a function of temperature with the use of a jacketed $20-\mathrm{cm}$. cell and a Zeiss $0.01^{\circ}$ polarimeter.

The influence of polymer concentration and of solvent composition on the transition temperature $T_{c}$ and on the slope of the polarimetric transition curve is shown in Figures 3 and 4 . The optical rotation is expressed in values of $[\varphi]_{D}$, the molal residue rotation, defined as $\alpha_{\mathbf{D}}(c d)^{-1}$, where $\alpha_{\mathrm{D}}$ is the measured optical rotation in degrees, $c$ is the concentration in mole

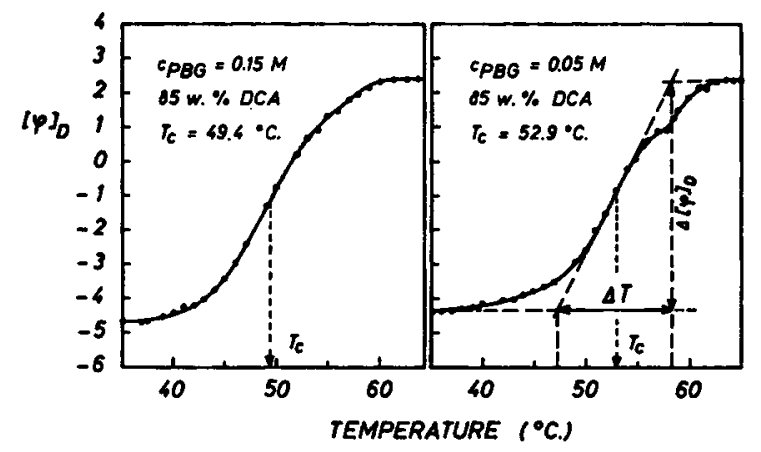

Fig. 3. Optical rotation as a function of temperature of two PBG solutions of different concentration, at constant solvent composition. See text for definition of $[\varphi]_{D}, \Delta[\varphi]_{D}$, and $\Delta T$.

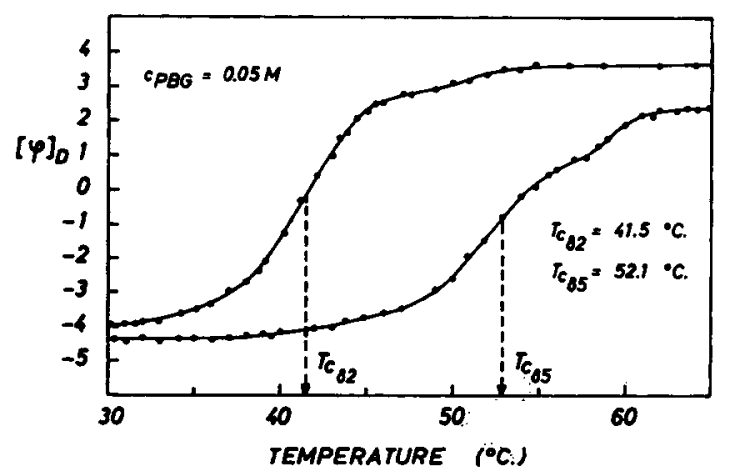

Fig. 4. Optical rotation as a function of temperature of two PBG solutions of equal concentration and of different DCA contents, 82 and 85 wt.- $\%$ DCA, respectively, with transition temperatures as indicated. 
residue/kilogram solvent, and $d$ is the length of the polarimeter tube in decimeters.

In order to interpret the polarimetric transition curves quantitatively, it is necessary to give values for the slope of such a curve at the transition temperature $T_{c}$, i.e., $\left(d[\varphi]_{\mathrm{D}} / d T\right)_{T_{c} \text {. }}$ In general, it is assumed that the dependence of $\theta$ on temperature corresponds to the temperature dependence of the optical rotation. ${ }^{15}$ Thus, for the midpoint of the conversion we can write

$$
(d \theta / d T)_{T_{c}}=(1 / \Delta T)_{T_{c}}
$$

(See the right part of Fig. 3, where $\Delta[\varphi]_{\mathrm{D}}$, the total change in optical rotation in going from coil to helix, corresponds to $\Delta \theta=1$.)

\section{Results}

In a previous study ${ }^{4}$ it was reported that the transition enthalpy $\Delta H$ at constant solvent composition is a linear function of the polymer concentration. The linear concentration dependence has been confirmed for further sets of measurements carried out with larger portions of DCA in the solvent mixture. Additionally, a strong dependence of $\Delta H$ on solvent composition was found.

The results of the calorimetric measurements with respect to $\Delta H$ are quantitatively summarized in Figure 5. The concentration lines become increasingly steeper for larger DCA/EDC ratios. However, the linear extrapolation leads in each case to nearly the same intercept at the ordi-

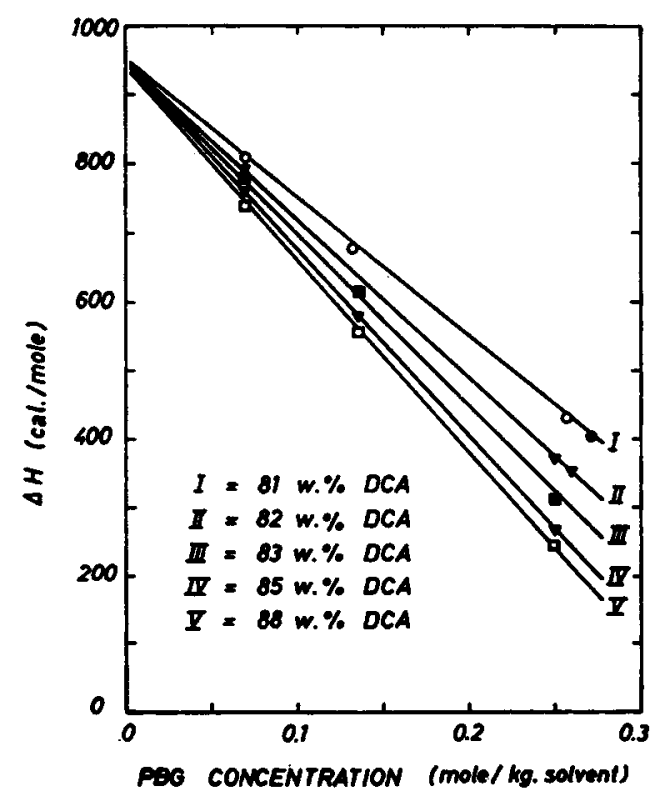

Fig. 5. Transition enthalpy $\Delta H$ as a function of $P B G$ concentration, at different solvent compositions: (O) data reported previously. 4 


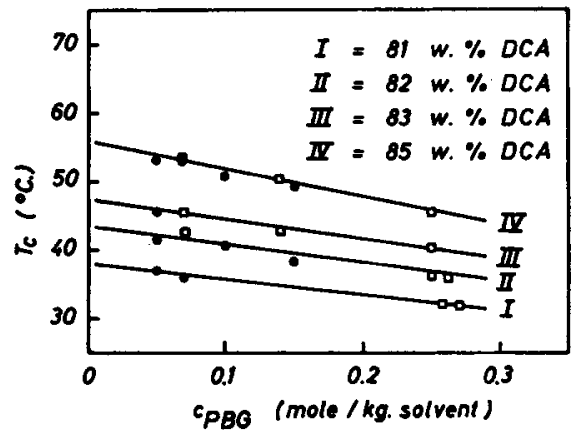

Fig. 6. Transition temperature $T_{c}$ as a function of PBG concentration at different solvent compositions: (๑) data obtained polarimetrically; ( $\square$ ) data obtained calorimetrically.

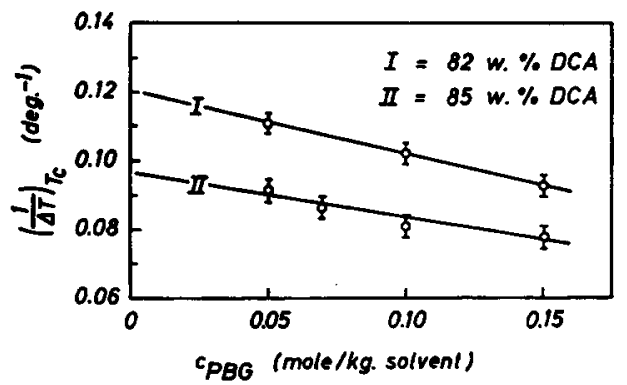

Fig. 7. Slope of polarimetric transition curve at the transition temperature expressed in terms of $(1 / \Delta T) r_{c}$ as a function of PBG concentration at two different solvent compositions.

nate. The physical meaning of this result is that, in the state of infinite dilution, there exists a standard value of the transition enthalpy:

$$
\Delta H^{0}=950 \pm 20 \mathrm{cal} . / \text { mole of amino acid residue }
$$

This standard value is independent of the solvent composition. The dependence of the $\Delta H$ values on the DCA content of the solvent mixture at given PBG concentrations is shown in Figure $8 a$.

It is shown in Figure 6 that, at constant solvent composition, the $T_{c}$ values obtained calorimetrically and polarimetrically fit well in the same linear relation between $T_{c}$ and the polymer concentration. The value of $T_{c}$ decreases with increasing PBG content of the mixture. For a given polypeptide concentration, $T_{c}$ is raised by an increase in the DCA weight fraction.

The slope of the polarimetric transition curve at $T_{c}$, expressed by (1/ $\Delta T)_{T_{c}}$ is observed to decrease with increasing concentration of the polypeptide at constant solvent composition. As shown in Figure 7, for constant polymer concentration, a rise in the DCA weight fraction is accompanied by a decrease in the slope value. A slight dependence on polymer 


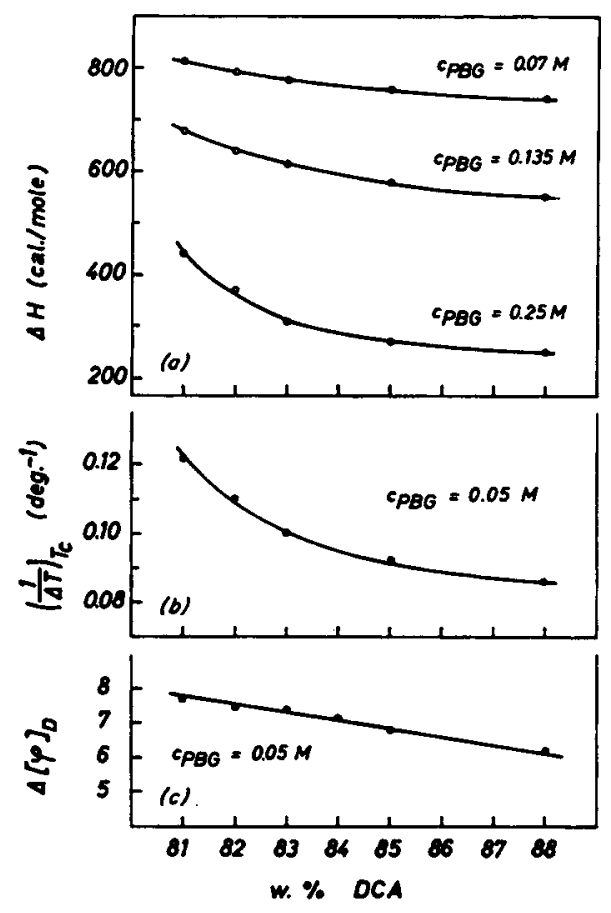

Fig. 8. Plots of (a) transition enthalpy $\Delta H$ (top), (b) slope of polarimetric transition curve at the transition temperature, expressed in terms of $(1 / \Delta T)_{T_{c}}$, and (c) total change of optical rotation in going from coil to helix, $\Delta[\varphi]_{D}$, all as a function of DCA weight fraction at PBG concentrations as indicated.

concentration was also found for the slope of the polarimetric transition curves by Karasz and $O^{\prime}$ Reilly in a study on poly-e-carbobenzoxy-Llysine. ${ }^{16}$

In Figure $8 b$, the slopes $d[\varphi]_{D} / d T$ at $T_{c}$ are plotted in terms of $(1 / \Delta T)_{T c}$ against the solvent composition. A qualitative similarity of this plot with the $\Delta H$ versus weight per cent DCA curves in the upper part of the figure is to be seen. The total change in the optical rotation in going from coil to helix, $\Delta[\varphi]_{\mathrm{D}}$, varies with the PBG concentration at constant solvent composition only within the margin of error, at least for the small values of $c_{\mathrm{PBG}}$ investigated in this study. However, at constant polymer concentration, $\Delta[\varphi]_{D}$ decreases linearly with increasing DCA content of the solvent mixture. This is shown in Figure $8 c$.

The value of the parameter $\sigma$ can be calculated [see eq. (2)] according to eq. (4):

$$
\sigma^{1 / 2}=(\Delta H)^{2} / 4 R T_{c}^{2} C_{c, \max }
$$

Thus, a simple means is provided by the theory to evaluate $\sigma$ from the course of the measured heat capacity data only in the temperature region of the transition. It follows from the calorimetric transition curves 


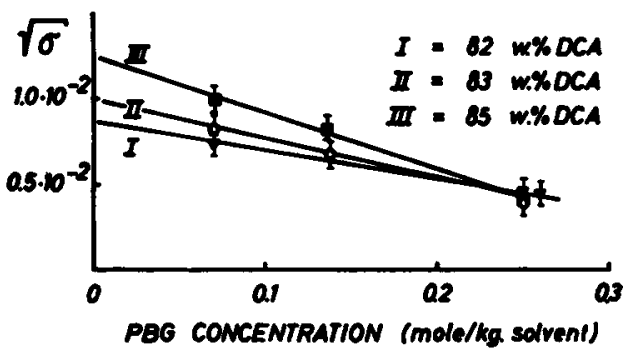

Fig. 9. Data of $\sigma^{1 / 2}$, calculated from eq. (4), as a function of PBG concentration at different solvent compositions.

that both $\Delta H$ and $C_{c, \max }$. decrease with increasing PBG concentrations. The use of eq. (4) for the determination of $\sigma^{2 / 2}$ leads to values depending on polymer concentration and on solvent composition, as shown in Figure 9.

If the $\Delta \boldsymbol{H}$ values are known, there is another method for obtaining $\sigma$, in which the results of two different techniques are combined. As mentioned in the foregoing section, it is generally assumed that the temperature dependence of the optical rotation corresponds to the $\theta$ versus $T$ curve. Applequist 12 has developed an equation describing the variability "of $\theta$ with temperature at $T_{c}$ :

$$
(d \theta / d T)_{T c}=\Delta H / 4 R T_{c}^{2} \sigma^{1 / 2}
$$

Thus, recalling eq. (3), $\sigma$ can be evaluated by use of eq. (5).

The so-called van't Hoff heat of transition, $\Delta H$ (van't Hoff), is correlated with the calorimetric transition enthalpy $\Delta H$ by eq. (6):

$$
\Delta H(\text { van't Hoff })^{\prime}=\Delta H \cdot \sigma^{1 / 2}
$$

The values of $\Delta H$ (van't Hoff) and of $\sigma$ calculated with use of the experimental data given in Figures 5-7 are summarized in Table $I$. The values of $\sigma^{1 / 2}$ obtained in this way show a similar dependence on polymer content and on solvent composition as those calculated according to eq. (4) (see Fig. 9).

\begin{tabular}{|c|c|c|c|}
\hline $\begin{array}{l}\text { DCA, } \\
\text { wt.-\% }\end{array}$ & $\begin{array}{c}c_{\mathrm{PBG}}, \\
\text { mole/kg. } \\
\text { solvent }\end{array}$ & $\begin{array}{c}\Delta H(\text { van't Hoff }) \\
\text { kcal./mole }\end{array}$ & $\sigma^{1 / 2} \times 10^{2}$ \\
\hline \multirow[t]{4}{*}{82} & 0.15 & 71.5 & 0.84 \\
\hline & 0.10 & 80.3 & 0.90 \\
\hline & 0.05 & 87.9 & 0.95 \\
\hline & $c_{\mathrm{PBG}} \rightarrow 0$ & 96.0 & 0.99 \\
\hline \multirow[t]{4}{*}{85} & 0.15 & 64.5 & 0.84 \\
\hline & 0.10 & 68.0 & 0.99 \\
\hline & 0.05 & 77.4 & 1.05 \\
\hline & $c_{P B G} \rightarrow 0$ & 84.1 & 1.13 \\
\hline
\end{tabular}

\section{TABLE I}




\section{Discussion}

It has been found experimentally that the data for the thermodynamic and optical properties characterizing the transition behavior of PBG in DCA-EDC are dependent on the mole fractions of the components. Most immediately, this is obvious for the transition temperature $T_{c}$ being a function of the polypeptide concentration as well as of the DCA weight fraction. The terms $T_{c}$ and $\Delta H$ are correlated in a thermodynamic equation derivable from relations given in the Zimm-Bragg theory.

In this statistical treatment of the helix-coil transition, two parameters, $\sigma$ and $s$, are introduced. The latter, $s$, can be thought of as the equilibrium constant for the incorporation into a helical section the first adjoining segment (the stoichiometric unit of the chain, i.e., the amino acid residue $-\mathrm{NH}-\mathrm{CO}-\mathrm{CHR}-$, is called a segment) from a random section of the polypeptide chain. ${ }^{\circ}$ The temperature at which $s$ is equal to unity is defined as the transition temperature $T_{c}$. Thus, the van't Hoff relation $\ln s=$ $\Delta S / R-\Delta H / R T$ is reduced to $T_{c}=\Delta H / \Delta S$, where $\Delta S$ is the conformational entropy change related to $s$.

For the state of infinite dilution (index zero) it follows,

$$
T_{c}^{\circ}=\Delta H^{\circ} / \Delta S^{\circ}
$$

As $\Delta H^{\circ}$ is independent of the solvent composition, a shift of $T_{c}$ to higher temperatures with increasing DCA/EDC ratio must be accompanied by a decrease in $\Delta S^{\circ}$.

From eqs. (3) and (5) we get eq. (8):

$$
(1 / \Delta T)_{T c}=\left(\Delta H / 4 R T_{c}{ }^{2}\right) \sigma^{1 / 2}
$$

Now, it might be expected from the observed changes in $(1 / \Delta T)_{T c}, T_{c}$, and $\Delta H$, as caused by variations in polymer concentration and solvent composition, that the values of $\sigma^{1 / 2}$ calculated from eq. (8) show the same dependence on $c_{P B G}$ and on DCA weight fraction as those resulting from $C_{c, \max }$, by eq. (4). At constant solvent composition, an increase in the polypeptide concentration causes a decrease in $\sigma^{1 / 2}$, evaluated from eq. (4) as well as from eq. (8), but at slightly different rates. On comparing Figure 9 and Table $I$ it is seen that the extrapolated $\sigma^{1 / 2}$ for infinite dilution are approximately the same, demonstrated for example in the systems of 82 and 85 wt.- $\%$ DCA.

In the standard state of infinite dilution differences in the extrapolated value of $(1 / \Delta T)_{T_{c}}{ }^{\circ}$ can only be caused by differences in the extrapolated values of $\left(\sigma^{1 / 2}\right)^{\circ}$ and of $T_{c}{ }^{\circ}$. If, for instance, the two systems with 82 and $85 \mathrm{wt} .-\%$ DCA are compared, according to eq. (8) the following relation is obtained:

$$
\frac{(1 / \Delta T)_{T_{c}}{ }^{\circ}(82)}{(1 / \Delta T)_{T c}{ }^{\circ}(85)}=\frac{\left(\sigma^{1 / 2}\right)^{\circ}(85)\left(T_{c}^{\circ}\right)^{2}(85)}{\left(\sigma^{1 / 2}\right)^{\circ}(82)\left(T_{c}{ }^{\circ}\right)^{2}(82)}
$$

This proportion is seen to be valid within $3 \%$ tolerance. 
Regarding finite concentrations of the polypeptide, differences in the slopes $(1 / \Delta T)_{T c}$ are essentially caused by changes in $\Delta H$ due to changes in the solvent composition. Therefore, at constant polymer concentration, the dependence of $\Delta H$ on the DCA weight fraction is very similar to that of $(1 / \Delta T)_{r_{c}}$ as shown in Figure 8. In the concentration region considered here the corresponding ratio of the slope values equals that of the $\Delta H$ data within the tolerance of $10 \%$. The data for $\Delta H$ and for $\sigma^{1 / 2}$, the latter evaluated from $C_{c, \max }$, at constant solvent composition, were found to be dependent on the PBG concentration. The fact that the $\Delta H$ (van't Hoff) values corresponding to these conditions show a concentration dependence a little different from that obtained for the calorimetric $\Delta H$ data must result in $\sigma^{1 / 2}$ values [calculated from eq. (8)] depending upon the polymer concentration (see Table I).

Thus, it has been shown that the experimental results concerning the dependence of the optical and thermodynamic properties on the polymer concentration and on the DCA content can be analyzed by using the same simple relation, eq. (5). Since the transition temperature $T_{c}$ is unequivocally determined by the PBG concentration and the content of DCA in the solvent, it is at least very unusual to describe the observed dependence of $\Delta H$ or even of $\sigma$ on the mole fractions of the components as a function of $T_{c}$, as done by Karasz and O'Reilly. ${ }^{11}$

The basic assumption of Zimm and Bragg that $\sigma$ is independent of temperature during the process of helix formation cannot be tested by the results reported in this paper. We only can say that, according to our experimental findings, $\sigma$ is dependent on the polypeptide concentration and, for relatively small contents of the polymer, also on the solvent composition of this system. However, in wide limits of PBG concentration and of DCA/EDC ratios, the values of $\sigma$ do not exceed the order of magnitude of $10^{-4}$.

In the discussion of Figure 1 it was mentioned that the heat capacity data below the transition region show a different course with temperature than those above. When the heat capacity versus temperature line below the transition region is extended to higher temperatures, it is seen that the heat capacity curve above the conformational conversion is lower, as would be expected of a helical conformation with relatively fewer degrees of freedom. However, it is not only a simple parallel shift. The plot of heat capacity above the transition region has a slope which is relatively smaller. Therefore it is not possible to give reliable values for $\Delta \bar{C}_{p}$, the partial molar heat capacity change in going from the randomly coiled to the helical conformation. Hence, a detailed thermodynamic interpretation according to Lumry et al. ${ }^{17}$ cannot be given. It should be also noted that it is the total PBG-solvent system which is involved in the structural conversion of the polypeptide. Therefore some caution is necessary in drawing conclusions from the experimental findings to properties concerning the polymer alone.

Recently, the possibility of protonation of the polypeptide amide group 
by dichloroacetic acid has been discussed. .8 $^{20}$ It is not yet very clear how and to what extent changes in protonation will influence the helix-coil transition of a polypeptide like PBG.

At the present state of our investigations on the PBG-DCA-EDC system it is not possible to give definite explanations for the observed dependences of $\Delta H$ and of $\sigma$ on both the polymer concentration and on the DCA content of the solvent. Possible models involving intermolecular interactions resulting in partial chain aggregation or in steric hindrances, especially in solutions of high polypeptide concentration, must be regarded as highly speculative. Measurements of optical rotatory dispersion and circular dichroism, for instance, offer possibilities to supplement the results obtained calorimetrically and polarimetrically and to find a useful model for theoretical considerations in this field.

\section{Conclusions}

The results of the calorimetric and polarimetric measurements can be expressed quantitatively to a satisfactory extent in terms of equations derived from the first-order approximation of the Zimm-Bragg theory. The helix-coil transition of poly ( $\gamma$-benzyl L-glutamate) represents a simple model reaction for the relatively complicated cooperative conversion of a polypeptide or a protein. The enthalpy change corresponding to the elementary process of helix formation in this PBG system is the transition enthalpy $\Delta H^{\circ}$, independent of the solvent composition.

The extrapolated values of $\Delta H$ and even of $\sigma$ agree very well with those estimated by use of the original theory ${ }^{\circ}$ and of its extensions. ${ }^{12,16,21}$ Nevertheless, the dependences of $\Delta H$ and of $\sigma$ on the polymer concentration and on the ratio of the solvent components must be taken into account when equations derived from the Zimm-Bragg theory are used, e.g., for calculating the fraction of helical residues or related properties.

\section{APPENDIX}

A minor peculiarity in the calorimetric and polarimetric transition curves is found at temperatures near the upper limit of the transition region. The heat capacity data show a small additional increase before following the linear course with $T$ in the temperature range above the transition region. This irregularity is reflected in the upper part of the corresponding optical rotation versus temperature curve, especially for solutions of low polymer cncentration (compare Figs. 1, 2, 3, and 4).

At higher temperatures, another peculiarity is observed in the course of the heat capacity with temperature. A second peak of the heat capacity data was found in the temperature range of $60-70^{\circ} \mathrm{C}$. for solutions of high polymer concentration (for instance, $c_{\mathrm{PBG}}=0.25 M$ ). The second peak appears between 70 and $80^{\circ} \mathrm{C}$. for solutions of low PBG concentrations (e.g., $\left.c_{\text {PBG }}=0.07 M\right)$, but only at relatively high DCA weight fractions. An example for the existence of such peculiarities is given in Figure 10. 


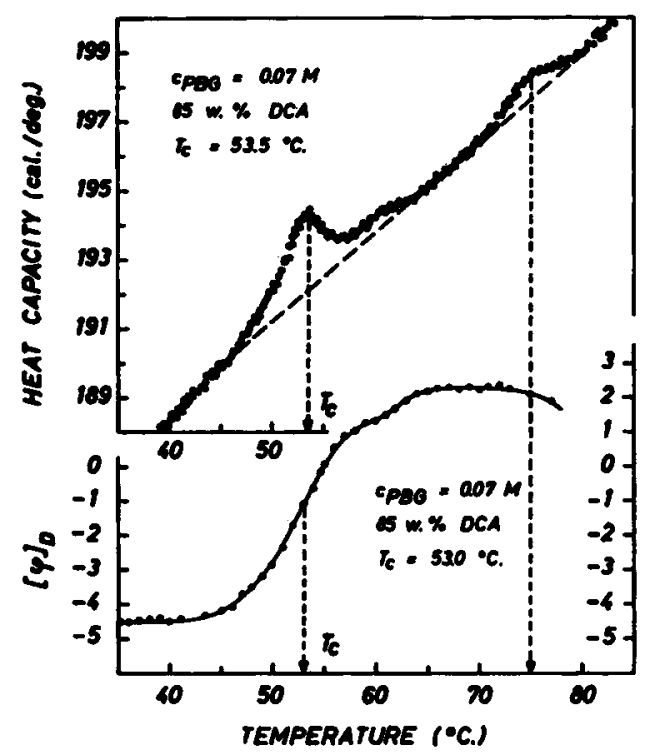

Fig. 10. Heat capacity (top) and optical rotation (bottom) of a PBG solution, both as a function of temperature.

It is seen that the course of the heat capacity data with temperature is reflected in that of the optical rotation. When the calorimetric measurement is repeated with the same solution, there is neither the first "normal" peak nor the second one in the course of the heat capacity data, but only a straight line. This result indicates, that, in the original measurement, the polymer has been denatured with respect to its primary structure during heating through the temperature region of the second peak. A change in the polymer structure at temperatures about $75^{\circ} \mathrm{C}$. is also reflected in the polarimetric transition curve which shows a fall.

For the planes under the second peak of the calorimetric transition curves, however, no simple dependence on polymer concentration and on DCA content was observed. Further investigations are intended in order to solve this new problem.

\section{References}

1. E. Katchalski and J. Z. Steinberg, Ann. Rev. Phys. Chem., 12, 433 (1961).

2. P. Doty and J. T. Yang, J. Am. Chem. Soc., 78, 498 (1956).

3. P. Urnes and P. Doty, Advan. Protein Chem., 16, 401 (1961).

4. Th. Ackermann and H. Rüterjans, Z. Physik. Chem. (Frankfurt), 41, 116 (1964).

5. F. E. Karass, J. M. O'Reilly, and B. E. Bair, Nature, 202, 693 (1964).

6. P. L. Privalov, J. R. Monaselidze, G. M. Mrevlishvili, and V. A. Magaldadze, Zh. Ekspll. 'Teor. Fiz., 47, 2073 (1964); Soviet Phys.JETP, 20, 1393 (1965).

7. P. L. Privalov, K. A. Kafiani, and D. R. Monaselidze, Dokl. Akad. Nauk SSSR, 156, 951 (1964).

8. Th. Ackermann and H. Rüterjans, Ber. Bunsenges. Physik. Chem., 68, 850 (1964).

9. B. H. Zimm and J. K. Bragg, J. Chem. Phys., 31,526 (1959). 
10. F. E. Karasz, private communication.

11. F. E. Karasz and J. M. O'Reilly, Biopolymers, 4, 1015 (1966).

12. J. Applequist, J. Chem. Phys., 38, 934 (1963).

13. Th. Ackermann, Z. Elektrochem., 62, 411 (1958).

14. Th. Ackermann, in Experimental Thermodynamics, Vol. I, J. P. McCullough, Ed., Butterworths, London, Chap. 12, in press.

15. B. H. Zimm, P. Doty, and K. Iso, Proc. Natl. Acad. Sci. U.S., 45, 1601 (1959).

16. F. E. Karasz, J. M. O'Reilly, and H. E. Bair, Biopolymers, 3, 241 (1965).

17. R. Lumry, R. Biltonen, and J. F. Brandts, Biopolymers, 4, 917 (1966).

18. H. Watanabe, K. Yoshioka, and A. Wada, Biopolymers, 2, 91 (1964).

19. H. Watanabe and K. Yoshioka, Biopolymers, 4, 43 (1966).

20. S. Hanlon and I. M. Klotz, Biochemistry, 4, 37 (1965).

21. S. Lifson and A. Roig, J. Chem. Phys., 34, 1963 (1961).

Received September 16, 1966

Revised March 2, 1967

Prod. No. B306 